\title{
Técnicas del ejercicio del poder interviniente en la formación del juicio profesional del auditor desde la perspectiva de Michael Foucault $^{1}$
}

\author{
Valderrama, Yosman ${ }^{2}$ \\ Universidad de los Andes, Venezuela \\ E-mail:yosmanv@hotmail.com \\ https://orcid.org/0000-0001-5250-7362
}

Recibido: 04 de mayo de 2020

Aceptado: 15 de junio de 2020

\begin{abstract}
Resumen
El poder interviniente en la formación del juicio profesional del auditor está representado por un conjunto de relacionamientos sociales que ejercen influencia sobre la decisión racional del contable, su efecto de dominación se desarrolla a partir de estrategias globalizadoras o cuantitativas e individualizantes o cualitativas. En este sentido, la presente investigación se centró en describir las técnicas del ejercicio del poder interviniente en la formación del juicio profesional del auditor desde la perspectiva de Foucault. El enfoque epistemológico adoptado lo fundamentó los postulados de Foucault (1988) desarrollado a partir de la interacción del investigador con siete (07) auditores profesionales en actividad, cuyo relato se obtuvo a través de la aplicación de historias de vida como técnica de investigación. Dentro de las consideraciones finales, el ejercicio del poder interviniente en la formación del juicio profesional del auditor se fortalecen la aplicación de técnicas de totalización, cuya esencia se enfoca en dirigir la conducta del auditor hacia campos específicos de una manera global; así como, en técnicas de individualización que ejerce una conducción particular del sujeto, y atiende parámetros que consideran la particularidad del auditor.
\end{abstract}

Palabras clave: Técnicas del ejercicio del poder, juicio profesional, Foucault.

1. Artículo de investigación derivado de la tesis doctoral titulada "Juicio Profesional del Auditor. Estrategias para su formación frente a la subjetividad impuesta por las relaciones de poder desde el enfoque de Michael Foucault", presentada ante la Universidad de Los Andes, Venezuela, posteriormente discutido y debatido en la XXX Conferencia Académica Permanente de Investigación Contable (CAPIC), Santa Marta, Colombia. 


\title{
Techniques of the exercise of the interventive power in the formation of the professional judgment of the auditor from the perspective of Michael Foucault
}

\begin{abstract}
The intervening power in the auditor's professional judgment formation is represented by a set of social relationships that exert influence over the rational decision of the accountant; its effect of domination is developed from globalizing or quantitative and individualizing or qualitative strategies. In this sense, the present investigation focused on describing the techniques of the exercise of power intervening in the formation of the professional judgment of the auditor from the perspective of Foucault. The epistemological approach adopted was based on the postulates of Foucault (1988) developed from the interaction of the researcher with seven (07) active professional auditors, whose story was obtained through the application of life stories as a research technique. Within the final considerations, the exercise of power intervening in the formation of the auditor's professional judgment strengthens the application of totalization techniques, whose essence is focused on directing the auditor's conduct towards specific fields in a global manner; as well as, in individualization techniques that exercises a particular conduction of the subject, and attends parameters that consider the particularity of the auditor.
\end{abstract}

Keywords: techniques of the exercise of power, professional judgment, Foucault.

\section{Introducción}

En el ámbito de esta investigación, el poder está caracterizado por el ejercicio de la dominación ejercida sobre el sujeto, la cual tiene influencia sobre la formación juicio profesional del auditor. Desde la óptica de Foucault (1988), este relacionamiento social representa "formas de explotación que separan a los individuos de aquello que ellos mismos producen; (...) que ata al individuo a sí mismo y lo subsume a otros" (p. 7). Por tanto, es un modo de autoridad en que las acciones de unos conducen o modifican las acciones de otros.

Foucault (1988) señala que los modos de dominación en la sociedad son el resultado de diversos procesos económicos y sociales que han dado lugar a diferentes relaciones de poder ejercidas sobre el sujeto, develándose de manera individual o en conjunto con otras; para este autor, algunas de ellas son "fuerzas de producción, lucha de clases y estructura ideológica que determinan formas de subjetividad" (p. 8).
Para Valderrama (2019) las relaciones de poder desde la perspectiva del juicio profesional del auditor, representan un orden de dominación que ejerce influencia sobre la formación del juicio y hacen que este se direccione hacia un campo específico, o lo conducen hacia la ejecución de actividades, es decir, es un escenario social que se presenta entre un campo que ejerce autoridad y la decisión racional del auditor que está subordinada a este.

En este contexto, las Normas Internacionales de Auditoría(NIA) (IAASB, 2018), develan un conjunto de relaciones de poder ejercidas sobre la formación del juicio profesional del auditor. Esta afirmación puede realizarse si se considera lo establecido en la NIA 200 (IAASB, 2018) la cual instituye que, al ejecutar una auditoría, el profesional contable deberá diseñar procedimientos y pruebas acatando los estándares internacionales emanados por la Federación Internacional de Contadores (IFAC, por sus siglas en ingles). Es decir, las NIA descubren una forma de dominación que incita al auditor a formar su juicio profesional a través del cumplimiento de 
un conjunto de parámetros y lineamientos.

Desde la óptica del poder de Foucault (1988), esta relación de sumisión ejercida por las NIA sobre el auditor, puede analizarse como un poder ético y social, que "ata al individuo a sí mismo y los subsume a otros" (p. 7), es decir, los preceptos normativos establecidos sobre el auditor evidencian un conjunto de relacionamientos que ejercen un orden de sumisión del profesional al ente emisor de normas; y un poder de sujeción, al motivar acciones específicas en la actividad profesional del auditor.

Entonces, las directrices de las NIA constituyen una relación de poder ejercida sobre el juicio profesional del auditor, considerando que establecen lineamientos y directrices bajo las cuales se debe llevar a cabo el ejercicio de la auditoría. Para Foucault (1988) este tipo de poder "no es una renuncia a la libertad, una transferencia de derechos, el poder de cada uno y de todos delegado a unos pocos" (p. 15), es decir, a partir del pensamiento foulcaultiano, es una relación de poder totalizante ejercida sobre el auditor.

Ahora bien, desde los postulados del Foucault (1988) los relacionamientos de poder presentes en la sociedad evidencian técnicas de una simultanea individualización y totalización, considerando por una parte, que diversas fuerzas de dominación se enfocan en la conducción del individuo para alcanzar un propósito específico; y por la otra, que "puede producir tanta aceptación al punto de ser deseado" (p. 15).

De esta manera, existen técnicas de poder globalizantes o totalizadoras, cuya esencia se centran en conducir al individuo dentro de una sociedad, a través de estrategias estandarizadas para todos los sujetos de una misma clase, así como, un poder individualizante cuyo orden de dominación establece un conjunto de diferenciaciones en el sujeto que lo hace actuar de modos pre establecidos, otorgando contextualizaciones a su actividad, las cuales, evidentemente ejercen dominación sobre la formación de su juicio profesional.

Ahora bien, describir las técnicas del ejercicio del poder interviniente en la formación del juicio profesional del auditor desde la perspectiva de Foucault (1988) es una necesidad para la profesión contable de la nueva era, considerando que para Valderrama (2020) es fundamental para el ejercicio pleno de la auditoría la aplicación de criterios independientes en la revisión de los estados financieros, lo cual es una demanda del entorno y de los interesados en la información financiera de las entidades.

Siendo así, se hace necesario promover a través de esta investigación la generación de nuevas formas de subjetividad, que impulsen al profesional contable a la liberación de las condiciones de dominación a las cuales históricamente ha sido sometido.

Estructuralmente el trabajo se desarrolló en tres apartados a saber: 1) fundamentos teóricos que denotan las técnicas del ejercicio del poder desde la perspectiva de Michael Foucault; 2) hallazgos de la investigación, obtenidos a partir de la interacción del investigador con los auditores en ejercicio profesional; y 3) algunas consideraciones finales enmarcadas en la descripción de las técnicas del dominación obtenidas de los hallazgos.

\section{Materiales y método}

En este estudio el investigador consideró los postulados teóricos de Foucault (1988) quien devela que, para el análisis del sujeto, es necesario interpretar el ejercicio del poder como un modo de acción sobre el comportamiento de otros, lo que hace que el poder este representado por relacionamientos sociales, en otras palabras, el sujeto se ve influenciado por otros en su forma de actuar, lo cual requiere de una ontología crítica.

La posición ontológica consideró al auditor en la formación de su juicio profesional, por cuanto es este quien emite los juicios con base en diversos aspectos, dentro de los cuales las experiencias y conocimientos tienen un rol fundamental en el racionamiento decisional emitido por el profesional.

El estudio consideró como sujetos interactuantes los auditores en actividad profesional, definido por 
el IAASB (2018) en el glosario de términos de las NIA como "la persona o personas que realizan la auditoría, normalmente el socio del encargo u otros miembros del equipo del encargo o, en su caso, la firma de auditoría " (p.16).

En atención a lo expuesto, se estimó pertinente acudir a siete (07) auditores en actividad profesional, con más de diez (10) años de experiencia en el ejercicio de auditorías de estados financieros; cuyo domicilio de trabajo se ubique en el estado Trujillo
- Venezuela, a fin de lograr la interacción social del investigador con dichos profesionales. Las características y condiciones de los entrevistados se detallan en la tabla 1.

La investigación se basó en el análisis del relato de los auditores, obtenido en el contexto donde forma los juicios en su actividad profesional, por tanto, se desarrolló la comprensión del poder ejercido sobre la formación del juicio del auditor, a la luz de las percepciones propias de estos profesionales,

Tabla 1. Características de los sujetos interactuantes.

\begin{tabular}{|c|c|}
\hline $\begin{array}{c}\text { Sujeto } \\
\text { entrevistado }\end{array}$ & Descripción \\
\hline Auditor 1 & $\begin{array}{l}\text { Hombre de } 41 \text { años, Técnico Superior Universitario en Administración (Fundación } \\
\text { La Salle), Licenciado en Contaduría Pública (UVM), Especialista en Gerencia } \\
\text { Tributaria (Universidad Valle del Momboy) (2012), Asesor Tributario y Auditor } \\
\text { desde el } 2007 \text {. }\end{array}$ \\
\hline Auditor 2 & $\begin{array}{l}\text { Hombre de } 48 \text { años, Técnico Superior Universitario en Banca y Finanzas (Instituto } \\
\text { Universitario de Tecnología del Estado Trujillo), Licenciado en Contaduría Pública } \\
\text { (Universidad Bicentenaria de Aragua), Especialista en Gerencia Tributaria } \\
\text { (Universidad Valle del Momboy), Magister en Gerencia Financiera (Universidad } \\
\text { Nacional Experimental Rafael María Baralt), Doctor en Educación (Universidad } \\
\text { Nacional Experimental Rafael María Baralt), Asesor Tributario, Profesor } \\
\text { Universitario y Auditor desde el 2002. }\end{array}$ \\
\hline Auditor 3 & $\begin{array}{l}\text { Mujer de } 53 \text { años, Técnico Superior Universitario en Administración de } \\
\text { Mantenimiento (Instituto Universitario de Tecnología del Estado Trujillo), } \\
\text { Licenciada en Contaduría Pública (UNA), Licenciada en Administración (UNA), } \\
\text { Especialista en Gerencia Tributaria (Universidad Santa María), Magister en } \\
\text { Ciencias Contables (Universidad de Los Andes), Asesor Tributario, Profesor } \\
\text { Universitario y Auditor desde 1997. }\end{array}$ \\
\hline Auditor 4 & $\begin{array}{l}\text { Hombre de } 64 \text { años, Licenciado en Contaduría Pública (Universidad de Los Andes), } \\
\text { Asesor Tributario y Auditor desde } 1978 .\end{array}$ \\
\hline Auditor 5 & $\begin{array}{l}\text { Hombre de } 60 \text { años, Licenciado en Contaduría Pública (Universidad de Oriente), } \\
\text { Actualmente cursando la Especialidad en Auditoría (Universidad del Zulia), Asesor } \\
\text { Tributario y Auditor desde } 1991 .\end{array}$ \\
\hline Auditor 6 & $\begin{array}{l}\text { Hombre de } 52 \text { años, Licenciado en Contaduría Pública (Universidad de Los Andes), } \\
\text { Licenciada en Administración (Universidad de Los Andes), Especialista en } \\
\text { Gerencia Empresarial (Universidad Rafael Urdaneta), Magister en Administración } \\
\text { (Universidad Valle del Momboy), Asesor Tributario y Auditor desde } 1992 .\end{array}$ \\
\hline Auditor 7 & $\begin{array}{l}\text { Mujer de } 47 \text { años, Licenciada en Contaduría Pública (Universidad del Zulia), } \\
\text { Especialista en Derecho Tributario (Universidad Santa María), Especialista en } \\
\text { Educación (Universidad Nacional Abierta), Magister en Educación Superior } \\
\text { (Universidad Rafael María Baralt), Magister en Gerencia Financiera (Universidad } \\
\text { Rafael María Baralt), Magister en Contaduría. Mención Auditoría (Universidad } \\
\text { Centro occidental Lisandro Alvarado), Doctor en Educación (Universidad Rafael } \\
\text { María Baralt), Asesor Tributario y Auditor desde hace más de } 22 \text { años. }\end{array}$ \\
\hline
\end{tabular}

Fuente: Elaboración propia (2019) 
permitiendo al investigador, apartarse de prejuicios o convicciones anticipadas, que vistas desde los postulados foucaultianos ejercen influencia sobre la individualidad del sujeto.

Además de esto, el trabajo consideró el análisis del sujeto (auditor) emisor de juicio en su propio contexto y cotidianidad, fundamentado en Foucault (1994) y sus argumentos propios que aluden que la comprensión del poder demanda la eliminación de teorías anticipadas a su interpretación, estas que pudieron formarse a partir de la existencia de la constitución del individuo como sujeto social, en sus diferentes prácticas de poder y sus incesantes juegos de verdad y sumisión.

Se realizó un acercamiento con los auditores a partir del diseño de estrategias particulares fundamentadas en evitar rechazos, respuestas inconclusas y otros elementos que perturbaran la comunicación, para Martínez (2008) estudios de esta naturaleza requieren concebir "el método y todo el arsenal de medios instrumentales como algo flexible, que se utiliza mientras resulta efectivo, pero que cambia de acuerdo con el dictamen" (p.146).

Desde esta perspectiva, el estudio aplicó como técnica de investigación las historias de vida, definidas por Rojas (2010) como una forma de construcción de la historia sobre el propio relato del sujeto; es decir, el estudio acudió a esta técnica como medio de acercamiento a los auditores.

En cuanto a la técnica de comprensión de los relatos, el investigador acudió a la aplicación de la interpretación de los mismos desde su propia percepción y subjetividad, descartando así la aplicación de software especializados para esta tarea de investigación.

\section{La totalización como técnica para el ejercicio del poder}

Foucault (1988) señala que las relaciones de poder desde la perspectiva totalizante "puede producir tanta aceptación al punto de ser deseado" (p. 15), esta evidencia se puede encontrar en opiniones de auditores profesionales entrevistados por el investigador quienes desde una óptica práctica exteriorizan su consentimiento por adoptar las NIA en su actuación, justifican su aplicación en diversas afirmaciones, dentro de las cuales destacan que las NIA representan la evolución de la actividad profesional del auditor hacia la estandarización de sus procedimientos; y que este cuerpo normativo es necesario, basándose en su aplicación y su comprensión mundial, lo que facilita la confiabilidad de la opinión del auditor expresada en el dictamen.

Atendiendo a esto, desde la perspectiva de Foucault (1988), podría firmarse que las técnicas de totalización ejercidas por las NIA no representan una renuncia a la libertad del auditor, sino que éste en su consentimiento, decide su adopción y manifiesta conformidad con su aplicación. El referido autor expresa, que un poder totalizante se ejerce cuando "no atiende solamente a la comunidad en su globalidad, sino a cada individuo en particular durante su vida" (p. 9).

Lo que quiere decir, que la relación de poder que emana desde las NIA distingue: por una parte, la institucionalización gremial, que ejerce autoridad en el ejercicio profesional del auditor; y por la otra, la individualidad del sujeto, influenciada por factores externos vinculados a la experiencia, la cual tiene su afectación en ideologías políticas, sociales, culturales y religiosas respectivas a la personalidad.

Así mismo, la relación de poder totalizante, develada por las NIA distingue motivaciones sociales, que han dado lugar a la creación de estándares internacionales, y han permitido unificar los criterios del auditor y los procedimientos de auditoría aplicados en el examen de la información financiera. Para Rancilla et al. (2011) los escándalos financieros, la pérdida de la credibilidad del auditor, entre otros, fueron los fundamentos base para la creación de Normas Internacionales de Auditoría. Por su parte, para el IAASB (2018) dentro de los fundamentos base para la creación de la normativa ha estado servir al interés público, el fortalecimiento de la profesión contable en el ámbito mundial y contribuir al desarrollo de las economías nacionales fuertes.

Sean escándalos financieros, crisis de la confianza 
o la atención del interés público, los hechos que motivaron la creación de las NIA, indiscutiblemente, corresponden a la disertación de Foucault (1988) cuando señala que las relaciones de poder son las respuestas actuales a sucesos sociales pasados.

Siendo así, la relación de sujeción totalizante, ejercida por el cuerpo normativo profesional sobre el juicio del auditor, distingue dos elementos clave identificados por Foucault (1988): "el otro", representado por el auditor, sobre el cual es ejercido el poder; y "un campo entero de respuestas, reacciones, resultados y posibles invenciones que pueden abrirse, el cual está enfrentando a una relación de poder" (p. 15), lo que cristaliza la esencia de la dominación e influencia sobre el sujeto.

Es de resaltar que la normativa profesional no representa la única fuerza de dominación totalizante ejercida sobre el juicio profesional del auditor, si se considera lo establecido en la NIA 200 (IAASB, 2018) cuando señala que la conducción de una auditoría deberá cumplir además de las NIA, con las disposiciones legales y regulatorias establecidas en cada país, evidenciando así una sujeción del auditor a las leyes, normativas y a las instituciones (Estado o gremios, como entes emisores de leyes y normas profesionales), las cuales establecen condiciones para actuar en la sociedad, y ejecutar la auditoría, así como, para diseñar los procedimientos necesarios para obtener evidencia suficiente y apropiada.

En este sentido, Kelsen (1988), expresa que "cada norma jurídica obliga a determinados seres humanos a observar, en ciertas circunstancias, una conducta determinada" (p. 3), originando una relación de poder entre los preceptos regulatorios y la formación del juicio profesional del auditor. Para Foucault (1988) esta relación de poder "consiste en guiar la posibilidad de conducta y poner en orden sus efectos posibles. Básicamente el poder es más una cuestión de gobierno" (p. 16).

De la visión de Kelsen (1988) se interpreta que estos efectos de gobernanza sobre el individuo muestran que la legislación y la normativa establecida sobre el ejercicio profesional del auditor dejan ver una relación de poder de acuerdo a los planteamientos de Foucault (1988), y en la que se identifican "sujetos individuales o colectivos que están enfrentados a un campo de posibilidades en el cual diversas formas de comportarse, diversas reacciones y comportamientos pueden ser realizados" (p. 16), promoviendo una autoridad de las instituciones sobre el auditor, e identifican dos sujetos involucrados: el dominante (Estado o gremio) y el sujeto a este (el auditor).

Desde los planteamientos de Foucault (1988), se compilan los actores reconocidos en una relación de poder; identificando, en primer lugar, un sujeto que ejerce autoridad, y en segundo, otro que está subordinado a ella. Lo que permite evidenciar a su vez dos relaciones de poder: por una parte, la normativa, y por otra, la legislación, que vistas desde los planteamientos del referido autor denotan un subordinado sobre el que se ejerce el poder, y un conjunto de leyes y normativas que estructuran el posible campo de acción del mismo, ejerciendo influencia en su juicio profesional.

De esta manera, la gobernanza normativa y legislativa ejercida sobre el sujeto evidencia órganos reguladores como la IFAC, así como, las instituciones del Estado, las cuales establecen los criterios para el ejercicio del auditor, o formas como debe comportarse este con su entorno, cuyos planteamientos tienen sus implicaciones en la formación del juicio profesional. Este tipo de poder generado por la gobernanza fue definido por Foucault (1988) como "un modo de acción que no opera directa o inmediatamente sobre los otros" (p, 15), desde esta perspectiva ejerce influencia de manera sutil sobre sus decisiones y juicios importantes.

Ante la conceptualización de Foucault (1988), la gobernanza como forma de dominación distingue dos condiciones: autonomía y totalización; juntas identifican una relación de poder a partir de las consideraciones foucaultianas; la primera, está representada por la libertad del auditor para acatar las imposiciones normativas establecidas sobre su ejercicio profesional; y la segunda, evidenciada en la subordinación, al adoptar estándares y lineamientos globales para el ejercicio pleno de la auditoría, todo esto con incidencia en el juicio profesional. 
De esta manera, la gobernanza instaurada sobre el ejercicio profesional del auditor influye de manera directa sobre la formación de su juicio profesional, lo cual instituye un adoctrinamiento normativo y legislativo impuesto sobre la profesionalidad del sujeto, cuyas condiciones de autonomía son el sustento sobre el cual se fortalece la dominación global ejercida a través de mecanismos reguladores enfocados en direccionar la conducta del individuo, y por tanto, la actividad profesional del auditor.

\section{Individualización como técnica para el ejercicio del poder}

Foucault (1988) señala que la individualización como técnica para el ejercicio del poder está definida como una fuerza de dominación enfocada en la conducción del individuo para alcanzar un propósito específico, es un orden autoritario emergente que somete al sujeto a través del direccionamiento de su conducta. Históricamente orientado a la dirección del hombre hacia la salvación de su alma, de allí su argumentación como poder pastoral.
Desde la perspectiva de las NIA (IAASB, 2018), el poder individualizante en la formación del juicio profesional del auditor se cristaliza en la evolución del gobierno de la globalidad, representando una forma de poder que transciende el propósito de conducir las almas hacia la salvación, para asegurar que la actividad profesional del auditor atienda el requerimiento de satisfacer el interés público y la atención de los mercados globales.

Para Foucault (1988) las relaciones de poder, además de la dominación ejercida por las instituciones sociales o sus leyes, engloban las fuerzas de control que ejerce una sociedad; es decir, comprende las distintas expresiones dominantes sobre los diferentes niveles, apoyándose mutuamente de manera sutil.

De este modo, los relacionamientos de dominación ejercidos por las instituciones y las leyes sobre la formación del juicio profesional del auditor, no solo establecen condiciones de poder globalizadoras, sino imponen escenarios de

Tabla 2. Matriz de relación de poder desde la estructura legislativa y normativa.

\begin{tabular}{|c|c|c|}
\hline \multirow{2}{*}{ Órgano } & \multicolumn{2}{|c|}{ Ejercicio del poder } \\
\hline & Totalizante & Individualizante \\
\hline $\begin{array}{l}\text { Estado: } \\
\text { Ejerce su poder en la } \\
\text { representación de } \\
\text { instituciones y } \\
\text { organizaciones públicas. }\end{array}$ & $\begin{array}{l}\text { Emisión de directrices de manera } \\
\text { coercitiva en forma de leyes, } \\
\text { decretos, providencias y } \\
\text { resoluciones, con el propósito de } \\
\text { controlar la conducta del individuo } \\
\text { en la sociedad. }\end{array}$ & $\begin{array}{l}\text { Atiende los preceptos } \\
\text { legales establecidos por el } \\
\text { Estado, los cuales sustentan } \\
\text { y regulan su conducta en la } \\
\text { sociedad. }\end{array}$ \\
\hline $\begin{array}{l}\text { Emisores de normas: } \\
\text { Amparado en la normativa } \\
\text { profesional ejerce dirección } \\
\text { sobre las actividades del } \\
\text { contador en ejercicio de las } \\
\text { funciones establecidas por la } \\
\text { Ley. }\end{array}$ & $\begin{array}{l}\text { Emana reglas y lineamientos } \\
\text { profesionales que deben ser } \\
\text { acatados por los contadores en el } \\
\text { ejercicio de sus funciones. Tal es el } \\
\text { caso de las Normas Internacionales } \\
\text { de Auditoría emitidas por la IFAC. }\end{array}$ & $\begin{array}{l}\text { Realiza su labor conforme a } \\
\text { las disposiciones } \\
\text { establecidas por los órganos } \\
\text { reguladores, y en atención a } \\
\text { ello actúa de acuerdo a sus } \\
\text { parámetros. }\end{array}$ \\
\hline
\end{tabular}

Fuente: Elaboración propia (2019), con base en el análisis de Foucault (1988). 
individualidad para la conducción del auditor como sujeto en la sociedad, del cual se espera además de un acatamiento normativo y legislativo, una distinción doctrinal cuyos aspectos característicos tienen incidencia en la formación de su decisión racional. Estas afirmaciones son estructuradas desde la posición del investigador en la matriz 1.

Como se evidencia en la matriz anterior, la dominación ejercida sobre la formación del juicio profesional del auditor, trasciende el poder totalizante establecido por las disposiciones regulatorias, la normativa y las instituciones, y se ubica en el plano de la individualidad, lo cual adicionalmente puede ser evidenciado en lo establecido por la NIA 200 (IAASB, 2018) apartado A25, la cual instituye que "la aplicación del juicio profesional en un caso concreto se basa en los hechos y en las circunstancias que el auditor conoce " ( $p, 95)$, es decir, los hechos y circunstancias interpretados por el auditor fundamentan la formación de su juicio profesional.

Las NIA (IAASB, 2018) develan la existencia de hechos y circunstancias que representan relaciones de poder sobre la formación del juicio profesional del auditor. Interpretando los lineamientos establecidos en la NIA 200 (IAASB, 2018) podría afirmarse que las decisiones, expectativas y actuaciones estarán limitadas o sujetas al conocimiento de cualquier hecho y circunstancia que se presente antes o durante el proceso de auditoría de la información financiera.

En cuyo razonamiento, podría considerarse que los hechos develados en el Manual del Código de Ética para Profesionales de la Contabilidad (IESBA, 2018), descubren una relación de dominación individualizante al explicar que el profesional contable está expuesto a: conflictos de interés, regalos e invitaciones de clientes, intereses financieros, evaluación inadecuada de los resultados, afinidad significativa con su cliente, posicionamiento de la entidad auditada a través de la pérdida de la objetividad y disuasión de actuar al margen de la normativa por presiones externas.

Estos hechos desde la óptica del Manual del Código de Ética para Profesionales de la Contabilidad
(IESBA, 2018), representan amenazas originadas por una diversidad de relaciones, que podrían comprometer el cumplimiento de los principios éticos requeridos en la actuación del profesional contable y, por tanto, afectan su independencia. Es decir, el referido código, reconociendo este conjunto de circunstancias, establece una variedad de salvaguardas para ser aplicadas por el profesional, lo cual evidencia hechos sociales que ejercen un poder individualizante sobre la formación del juicio profesional del auditor.

Foucault (1988) expresa que las relaciones de poder en una sociedad establecen un "sistema de diferenciaciones, que permite actuar sobre la acciones de otros" ( $p, 18)$, de esta manera, la capacitación profesional establece un conjunto de características particulares entre cada individuo, otorgando estatus en el conocimiento adquirido; la influencia de padres, maestros y otros agentes de la sociedad, podrían agregar rasgos individuales en cuanto a valores y principios, que desde la postura foucaultiana sugieren que "cada relacionamiento de poder pone en funcionamiento diferenciaciones que son al mismo tiempo sus condiciones y sus resultados" (p. 18).

De esta manera, el poder individualizante como orden de dominación ejercido sobre la formación del juicio profesional del auditor establece un conjunto de diferenciaciones en el sujeto que lo hace actuar de modos pre establecidos, otorgando un conjunto de contextualizaciones a su actividad, que evidentemente ejercen dominación sobre la formación de su juicio profesional.

\section{EI relato de los entrevistados}

Con base en el relato de los auditores entrevistados se puede evidenciar dos técnicas puntuales para el ejercicio de la dominación cuyos efectos tienen incidencia directa en la formación del juicio profesional del auditor, las cuales, en concordancia con los postulados de Foucault (1988) son: técnicas individualizante y totalizante.

Las relaciones de poder ejercidas sobre la formación del juicio profesional del auditor 
develan, técnicas de individualización, al establecer condiciones particulares que imponen características propias a cada sujeto, para Foucault (1988) esta forma de subjetividad "no es meramente una forma de poder que ordena" (p.9), sino que este ejerce influencia sobre el sujeto de una manera seductiva y persuasiva, que impulsa al auditor a actuar de diferentes modos e incluso a determinar su comportamiento y acción con base en un precepto, sea este político, social o religioso.

Esta técnica de poder individualizante puede evidenciarse en el análisis de las fuerzas de subjetividad, que imponen particularidades a cada sujeto y este en su libertad permite la influencia de su comportamiento y su juicio, al respecto el Auditor 1 expresó:

resulta que yo era jefe de personal a los 15 años, entonces yo me involucre más hacia la parte laboral, desde muy temprana edad, pero nunca había visto la parte contable hasta ese momento que me tocó enfrentar una situación conflictiva, a partir de ahí comencé en el estudio la contabilidad.

Siendo así, la subjetividad impuesta por situaciones conflictivas instaura un orden de dominación ejerciendo un poder individualizante sobre el auditor, el cual desarrolla su actividad profesional hacia caminos específicos, denotando una relación de dominación con incidencia clara en la formación del juicio profesional del auditor.

En lo concerniente a la técnica totalizante el Auditor 1 manifestó que "la norma internacional pide que el contador sea formado en Auditoría", en concordancia, el Auditor 4 expresó que "las normas son prácticamente los principios... son los parámetros o estándares que están establecidos y que uno tiene que estar actualizado para saber cómo desarrollar una auditoría".

Significa entonces, que los estándares internacionales en el ámbito de auditoría establecen, por una parte, que la formación profesional del contador se debe enfocar en la actividad de auditoría; y por la otra, instituyen principios generales para la realización del trabajo. De tal modo que la normativa evidencia estrategias globalizadoras de adoctrinamiento profesional, a través de la imposición de parámetros generales que impulsan acciones conductistas para la formación del juicio del auditor durante su labor profesional.

Desde la perspectiva de Foucault (1988), este hallazgo de investigación podría denotar un establecimiento de metas a través del direccionamiento del individuo, que impone lineamientos específicos de dominación cuantitativa por cuanto no considera al individuo en su particularidad, sino atiende a una estrategia general de conducción del sujeto hacia la sociedad.

Dado los hallazgos de la investigación, la técnica poder totalizante puede evidenciarse en las diferentes luchas desarrolladas contra la sujeción, las cuales, de conformidad con las historias de vida de los auditores se oponen al adoctrinamiento normativo y social; así mismo, en las luchas contra la sumisión, sediciosas del direccionamiento de la actividad profesional.

Los Auditores 1, 2, 4, 5, 6 y 7 develan que la dominación caracterizada por el adoctrinamiento normativo impulsa un orden de sujeción totalizante, particularmente el Auditor 2, al ser abordado con la interrogante: ¿usted considera de las normas profesionales enmarcadas dentro del ámbito de la auditoría influyen en su juicio profesional?, respondió textualmente lo siguiente; "si porque ellas tienen unos lineamientos a nivel mundial", es decir, desde el planteamiento del referido auditor, el orden de dominación ejercido por las normas profesionales establecidas sobre la auditoría ejercen una dominación globalizadora que impulsa al auditor a comportarse y ajustarse a condiciones específicas.

El propio relato del Auditor 2 expresa "uno no tiene un juicio específico, uno tiene que ver cómo hacer para acomodarlo para amoldarlo a que encuadre en cada uno de esos aspectos que están allí en las normas", en otras palabras, la sujeción ejercida por la normativa profesional es una forma de poder totalizante desarrollado 
por encima de las condiciones del individuo, pero a su vez, individualizante, cuando impone restricciones propias a sujetos particulares.

Para Foucault (1988) las relaciones de poder que presentan las características mencionadas constituyen una evolución de la dominación, la cual "es una forma de poder que no sólo se preocupa por toda la comunidad, sino por cada individuo particular" (p, 9); es decir, es una forma de poder pastoral que atiende el ámbito cuantitativo o globalizador y a su vez subjetivo e individual.

En este contexto, el poder individualizante ejercido sobre la formación del juicio profesional del auditor impulsó desde los planteamientos de Foucault (1988) el descubrimiento de dos aspectos básicos; el de la institucionalización, representado en el Estado como jerarca legislativo, los órganos reguladores y emisores de normas, así como, la gremialización profesional; y el de la función de las instituciones, la cual cimienta el ejercicio de la dominación a través del direccionamiento del individuo y del establecimiento de mecanismos de control, seducción e imposición que buscan conducir al auditor hacia campos específicos, limitando su libertad, y en ocasiones, violando la autonomía propia del sujeto. El Auditor 4 sobre este aspecto señaló:

las normas nos hablan de algo, es decir, la oferta del servicio, que si la carta de representación, que si la carta de responsabilidad todo lo que tú tienes que lograr previo al cliente, para tu poder tener un cliente tú tienes que tener una conversación, tú tienes que hacer una oferta de servicio.

Con base en estos argumentos, la normativa profesional además de ser conductista, desarrolla relaciones de poder a través de un enfoque globalizador y colectivo (totalizante), atiende a principios de universalidad en la actividad profesional del auditor, pero a su vez incide en la individualidad del sujeto, al imponer condiciones previas o mecanismos de acción que deben seguirse en el desarrollo de una auditoría, lo cual de una manera $u$ otra, representa una intromisión a la voluntad del profesional, al instaurar mecanismos propios en el desarrollo de la actividad auditora.

Finalmente, las técnicas del ejercicio del poder interviniente en la formación del juicio profesional del auditor se fortalecen en el desarrollo de la totalización, al intentar dirigir la conducta del auditor hacia campos específicos de una manera global, la cual es cuantitativa al no considerar la particularidad del sujeto; así mismo, aplica la individualización a partir de la conducción particular del auditor, es decir, atiende parámetros que consideran la particularidad del individuo fomentándose así una técnica cualitativa.

\section{Conclusiones}

Los factores externos al auditor, tales como: las reglamentaciones en materia contable, la presencia de órganos reguladores, el contexto social y económico vinculante a su profesionalismo, las ideologías culturales, políticas y religiosas, los valores, representan una subjetividad social que interviene en la formación del juicio del auditor; conformando su perspectiva particular sobre la aplicación de procedimientos de auditoría, o la naturaleza, extensión y oportunidad de ellos, influenciado por su modo de interpretación de la normativa a la cual está subyugado.

Siendo así, las relaciones de poder presentes en la formación del juicio profesional del auditor trascienden el campo de las leyes, normas e instituciones, se conducen por un complejo campo de ideologías culturales, políticas y religiosas, y transitan por el lenguaje, los valores, y el conocimiento, representando una subjetividad social que interviene en la formación del juicio profesional del auditor.

Significa entonces, que la subjetividad social es un factor determinante en la formación del juicio profesional del auditor, por cuanto para Foucault (1988) el poder que ejerce la sociedad en el individuo ha hecho que su pensamiento y sus características habituales cambien de uno a otro, derivado de los procesos económicos y sociales, 
fuerzas de producción, luchas de clase y estructuras ideológicas.

De esta manera, las relaciones de poder en la sociedad representan una forma de vivir día a día, cuyas condiciones básicas de subsistencia recaen en la libertad, y la acción de influir sobre otros. Su reconocimiento no pretende sostener que estas son una necesidad para la sociedad, es argumentar desde la perspectiva de Foucault (1988) que su análisis es ineludible, para crear medios que contribuyan al individuo en su lucha contra la sujeción, subjetividad y sumisión a la que ha sido sometido, y cuyas condiciones han marcado su propia individualidad.

\section{Referencias bibliográficas}

Foucault, M. (1988). El sujeto y el poder. Edición electrónica de http://www.philosophia. cl/biblioteca/Foucault/E1\%20sujeto $\% 20$ y\%20el\%20poder.pdf Escuela de Filosofía. Universidad ARCIS.

Foucault, M. (1994). Hermenéutica del Sujeto. Edición y traducción: Fernando ÁlvarezUría. Libro el Línea. Disponible en: https:// seminarioatap.files.wordpress.com/2013/02/ foucault-michel-hermeneutica-del-sujeto.pdf (Consultado en 2018, febrero 10).

INTERNATIONAL AUDITING AND ASSURANCE STANDARDS BOARD (IAASB). (2018) Norma Internacional de Auditoría $\mathrm{N}^{\circ}$ 200, Objetivo y Principios Generales que gobiernan una Auditoría de Estados Financieros. Londres, Inglaterra.

INTERNATIONAL AUDITING AND

ASSURANCE STANDARDS BOARD (IAASB). (2018) Handbook of International Quality Control, Auditing, Review, Other Assurance, and Related Services Pronouncements. Londres, Inglaterra.

INTERNATIONAL ETHICS STANDARDS BOARD FOR ACCOUNTANTS (IESBA). (2018) Code of Ethics for Accounting Professionals. New York, USA.

Kelsen. H. (1988). Teoría general del derecho y el Estado. Universidad Nacional Autónoma de México. México. Traducción de Eduardo García.

Martínez, M. (2008). Epistemología y Metodología Cualitativa en las Ciencias Sociales. Trillas. México.

Ranzilla, S., Chevalir, R., Hermann, G., Glover, S. y Prawitt, D. (2011). Elevating Professional Judgment in Auditing and Accounting: The KPMG Professional Judgment Framework. Documento en Línea. Disponible en: https:// university.kpmg.us/content/dam/kpmg-univ/ prof-judgment/student/kpmg-prof-judgmentmonograph.pdf. (Consultado en 2016, mayo 12).

Rojas, B. (2010). Investigación cualitativa. Fundamentos y praxis. Segunda Edición. FEDUPEL. Caracas - Venezuela.

Valderrama, Y. (2019). Juicio profesional del auditor. Estrategias para su formación frente a la subjetividad impuesta por las relaciones de poder desde el enfoque de Michael Foucault. Tesis doctoral publicada. Universidad de Los Andes. Mérida, Venezuela.

Valderrama, Y. (2020). Condiciones históricas del poder interviniente en la formación del juicio profesional del auditor. Una interpretación desde Platón hasta Foucault. Revista Contabilidad y Auditoría. Disponible en: https://ojs.econ.uba. ar/index.php/Contyaudit/article/view/1730. (Consultado en 2020, noviembre 30). 